\title{
Narrative review of intraductal papillary mucinous neoplasms: pathogenesis, diagnosis, and treatment of a true precancerous lesion
}

\author{
Gang Ma, Guichen Li, Zhihuan Xiao, Anjiang Gou, Yuanhong Xu, Shaowei Song, Kejian Guo, Zhe Liu \\ Department of Pancreatic-Biliary Surgery, First Hospital of China Medical University, Shenyang, China \\ Contributions: (I) Conception and design: Z Liu, G Ma; (II) Administrative support: G Li, Y Xu, S Song; (III) Provision of study materials or patients: \\ A Gou, Z Xiao; (IV) Collection and assembly of data: G Li, A Gou, Z Xiao; (V) Data analysis and interpretation: G Ma, Z Liu; (VI) Manuscript \\ writing: All authors; (VII) Final approval of manuscript: All authors. \\ Correspondence to: Zhe Liu. Department of Pancreatic-Biliary Surgery, First Hospital of China Medical University, Shenyang 110001, China. \\ Email: liuzhecmu@126.com.
}

\begin{abstract}
Objective: Although considerable progress has been made in our understanding of intraductal papillary mucinous neoplasm (IPMN) of the pancreas, there are still some problems to be solved.

Background: IPMN is one of the most important precancerous lesions of pancreatic cancer, but the relationship between IPMN and pancreatic cancer, and the specific mechanism of the development from IPMN to invasive carcinoma, remain to be explored in depth. With the development of imaging, the detection rate of IPMN has been greatly improved. However, the degree of malignancy of IPMN is difficult to assess, and its classification criteria and surgical treatment strategies are still controversial. Therefore, there is an urgent need for the best treatment plan for IPMN and research that can better predict IPMN recurrence and tumor malignancy.

Methods: From the online database Web of Science (https://webofknowledge.com/) and PubMed (https:// pubmed.ncbi.nlm.nih.gov/), we use specific retrieval strategies to retrieve relevant articles based on the topics we discussed, and we review and discuss them.

Conclusions: This paper discusses the related research and progress of IPMN in recent years to improve the understanding of the incidence, diagnosis, treatment, and prognosis of this disease. The follow-up and monitoring of IPMN is particularly important, but the specific strategy also remains controversial.
\end{abstract}

Keywords: Intraductal papillary mucinous neoplasm (IPMN); invasive carcinoma; pancreatic cancer; surveillance; pancreas

Submitted May 25, 2021. Accepted for publication Jul 14, 2021.

doi: $10.21037 / g s-21-450$

View this article at: https://dx.doi.org/10.21037/gs-21-450

\section{Introduction}

It is estimated that by 2030, pancreatic cancer will become the second leading cause of cancer-related deaths in the United States (1), and is predicted to overtake breast cancer as the third leading cause of cancer-related deaths in the European Union (2). Most pancreatic cancers arise from the malignant evolution of pancreatic cystic neoplasms (PCNs). Pancreatic intraductal papillary mucinous neoplasm (IPMN) is a subtype of PCNs (3). It is an epithelial tumor, often originating in the main pancreatic duct or branch pancreatic duct, and is grossly visible, mainly showing papillary hyperplasia and having the function of secreting mucus. IPMN has always been considered a true primary pancreatic cystic precancerous lesion (4). It is one of the most important precancerous lesions of pancreatic ductal adenocarcinoma (PDAC), and approximately onethird of IPMNs are found to have associated invasive adenocarcinoma at the time of resection (5). IPMN is a 
mucin-producing tumor, which is characterized by the papillary proliferation of mucin-producing epithelial cells in pancreatic ducts and the secretion of large amounts of mucus. With the development of imaging, the detection rate of IPMN has increased significantly, but the assessment of its malignancy and surgical treatment are still controversial. This review summarizes the relevant research and progress on IPMN in recent years to improve the understanding of the incidence, diagnosis, treatment, and prognosis of this disease. We present the following article in accordance with the Narrative Review reporting checklist (available at https://dx.doi.org/10.21037/gs-21-450).

\section{Methods}

The research strategy involved the online databases Web of Science (https://webofknowledge.com/) and PubMed (https://pubmed.ncbi.nlm.nih.gov/). The search strategy included the following keywords: intraductal papillary mucinous neoplasm, IPMN, pancreatic, pancreatic tumor, pancreatic cancer, pancreatic ductal adenocarcinoma, mucinous cystic neoplasm, neuroendocrine tumors, pancreatic cystic tumors, diagnosis, epidemiology, histopathology, treatment, follow-up, guideline. Specifically, the research was restricted using variable combinations of the keywords. Articles in English published between January 1970 and April 2021 were searched. Some articles were excluded as they were not related to the topic of discussion.

\section{Incidence}

Researchers have found a variety of molecular genetic changes in IPMNs. Some of these genetic changes are similar to those found in PDAC, such as those of KRAS, $S M A D 4$, and TP53 genes, and the frequency range of $K R A S$ mutations in IPMNs is $40-81 \%$ (6). KRAS, $G N A S$, and RNF43 are the most common mutant genes of IPMN, and the point mutation of GNAS codon 201 is the most common and specific molecular phenomenon of IPMN (7). Other genes with recurrent somatic mutations in IPMN include $C D K N 2 A, C Y P 4 Z 1, D N A H 9, H L A-D Q B 2$, KIAA1109, MUC4, MUC12, PHF3, RBM10, RXFP2, SLC7A8, SLC9A3, ZNF260, and ZNF835 (8). GNAS mutations are common and unique to IPMN, and GNAS mutations are more common in intestinal subtypes, while $K R A S$ mutations are more common in pancreaticobiliary subtypes (9). Histological analysis and the high frequency of $K R A S$ mutations in IPMN indicate that oncogenic KRAS drives the development of IPMN in pancreatic duct cells, however, inducing the KRAS mutation in ductal cells does not produce IPMN. A study showed that IPMN can be the result of the synergistic effect of KrasG12D mutation and tumor suppressor gene inactivation (10).

A study also showed that, in the past few decades, the relative proportion of IPMN in PCNs has increased significantly. Whether this means that the incidence is indeed increasing or the detection rate is increasing is still controversial (11). There is no significant difference in the incidence of IPMN between men and women, though it may be that women have a slightly higher incidence than men (12). In 2004, a large study investigating pancreatic tumors and lesions found that in a series of 1,454 cases of tumorous lesions of the pancreas collected between 1971 and 2003, IPMN accounted for $18 \%$ of cysts, and was considered the most frequent cystic neoplasm (13). In a study of 30 university hospitals in South Korea from January 1993 to June 2005, out of 1,064 cases of PCNs confirmed by clinical pathological data analysis, a total of 436 cases were classified as IPMN (14). A multicenter research report in 2019 from India showed that IPMN accounted for $8 \%$ of operated patients with PCNs, but in China it accounted for $22 \%$ (15). Regarding the relationship between smoking and IPMN, there are different results in several studies. A 2014 study showed that positive smoking history does not seem to be a risk factor for high-grade dysplasia or invasive cancer in IPMNs (16). Another study in 2017 showed that current smoking is related to the incidence of PDAC concomitant with IPMN (17). A retrospective study published in 2016 showed that smoking may lead to the accelerated development of IPMN and the process of developing an aggressive malignant disease (18). A study have shown that the main pancreatic duct is the best predictor of high-grade dysplasia or invasive cancer in IPMN (19). The risk of IPMN progression will increase over time.

\section{Diagnosis and histopathology}

Early detection of IPMN is difficult, as the clinical manifestations of IPMN patients are non-specific and the onset is hidden. Some IPMN patients have no clinical symptoms at the time of diagnosis, and they are often discovered accidentally due to physical examination or other diseases through imaging examinations during diagnosis and treatment. A 2016 study from South Korea included 21,745 asymptomatic individuals who underwent 
Table 1 Three types of catheters through radiological findings by the Fukuoka International Consensus Guidelines

\begin{tabular}{ll}
\hline Names of types & Radiological findings \\
\hline MD-IPMN & Segmental or diffuse dilation of the MPD of $>5 \mathrm{~mm}$ without other causes of obstruction \\
BD-IPMN & Pancreatic cyst of $>5 \mathrm{~mm}$ in diameter that communicates with the MPD \\
Mixed-IPMN & Meets the criteria for both MD-IPMN and BD-IPMN \\
\hline
\end{tabular}

IPMN, intraductal papillary mucinous neoplasm; MPD, main pancreatic duct.

Table 2 "Worrisome features" and "high-risk stigmata" (25) of cysts

\begin{tabular}{|c|c|}
\hline Worrisome features & High-risk stigmata \\
\hline Thickened enhanced cyst walls & MPD size of $\geq 10 \mathrm{~mm}$ \\
\hline \multicolumn{2}{|l|}{ MPD size of $5-9 \mathrm{~mm}$} \\
\hline \multicolumn{2}{|l|}{ Lymphadenopathy } \\
\hline \multicolumn{2}{|c|}{ An elevated serum level of carbohydrate antigen (CA) 19-9 } \\
\hline A rapid rate of cyst growth $>5 \mathrm{~mm} / 2$ years & \\
\hline
\end{tabular}

abdominal CT scans as a health screening examination. Two radiologists evaluated the scan results and found 457 cases of occasional PCNs, and 376 cases were classified as IPMN (20). The general methods of diagnosing IPMN include carcinoembryonic antigen (CEA), amylase and molecular biomarkers, clinical history, gender, imaging characteristics, cytology, and cyst fluid analysis. The WHO's essential and desirable diagnostic criteria are as follows (21): (I) a grossly visible epithelial lesion within the pancreatic ductal system, with papillary formation; (II) may have gastric-type, intestinal-type, or pancreatobiliarytype epithelium; (III) associated invasive carcinoma must be excluded. The clinical diagnosis of IPMN mainly relies on imaging examinations, such as CT and MRCP, but histopathology is the final diagnostic gold standard.

IPMN is divided into main duct type (MD-IPMN), branch duct type (BD-IPMN), and mixed type (MTIPMN), according to the location of the lesion. MDIPMN, regardless of the presence of symptoms, has a high incidence of malignancy, while BD-IPMN, which is usually discovered accidentally, has a much lower incidence of malignancy $(11,22,23)$. MD-IPMN and MT-IPMN may be more symptomatic than BD-IPMN, and these
2 subtypes may be more related to malignant tumors. In all IPMN subtypes, the presence and duration of symptoms are related to a significant increase in the risk of malignancy $(23,24)$. After aggressive morphological changes, invasive carcinoma originating from branching ductal IPMNs is more aggressive (25). In 2016, the Fukuoka International Consensus Guidelines defined 3 types of catheters through radiological findings (26) (Table 1). The Fukuoka Guidelines also specifically explain that patients who have "worrisome features" on imaging should be evaluated by endoscopic ultrasonography (EUS) to further stratify the lesion, cysts with obvious "high-risk stigmata" should undergo resection without continued testing, and cysts of $\leq 3 \mathrm{~cm}$ in size without "worrisome features" should be monitored according to size stratification (26) (Table 2).

Magnetic resonance imaging (MRI) combined with magnetic resonance cholangiopancreatography (MRCP) is a better diagnostic method than computed tomography (CT) for IPMN, as a study showed that repeated exposure to ionizing radiation during CT increases the risk of malignancy (27). EUS is a powerful tool for diagnosing IPMN. It can clearly display the wall nodules in the expanded main pancreatic duct as well as the blood 
supply, effectively detect wall nodules and invasion, and describe malignant features (28). A multicenter research study showed that the ability of MRI-MRCP and EUS to detect worrisome features and high-risk stigmata in highly probable BD-IPMN had poor concordance (29). There is still debate as to whether MRI/MRI-MRCP or EUS is more accurate for diagnosing IPMN. In a retrospective study, MRCP was more effective than EUS for differentiating malignant from benign pancreatic IPMN and mucinous cystic neoplasm (MCN) (30). However, another study showed that there was no significant difference in the diagnostic performance of MRI and EUS in predicting malignant IPMN (31). A larger series of studies may be needed to compare EUS and MRI-MRCP.

Endoscopic retrograde cholangiopancreatography (ERCP) is also one of the methods used to diagnose IPMN. ERCP can clearly diagnose IPMN and distinguish between chronic pancreatitis and MPD expansion caused by IPMN, and can collect pancreatic juice. In a retrospective study of 138 IPMN patients in the same hospital, it was found that ERCP is more suitable for patients with MPD expansion and should not be used for patients with cysts larger than $30 \mathrm{~mm}$ (32). However, ERCP is not suitable as a routine examination method for IPMN, as it easily causes postoperative pancreatitis. In terms of inspection, it has a unique therapeutic effect in some cases. For example, when the patient's physical condition cannot tolerate the operation, and even some serious complications (obstructive jaundice, acute pancreatitis, etc.) occur, ERCP can treat or alleviate these complications while helping to confirm the diagnosis (Figure 1). Considering the high incidence of IPMN in the elderly, further application and development of ERCP in IPMN is worth further investigation.

In mucinous tumors, as mentioned above, GNAS mutations are unique to IPMN, hence, the analysis of GNAS positive mutations may be helpful in distinguishing IPMN and MCN (33-35). A total of 70\% of IPMN diseases occur in the head of the pancreas, $20 \%$ of IPMNs occur in the body or tail, and $5-10 \%$ are multifocal (36). MCNs are almost only found in women (37). In contrast to IPMN, MCN is a large mucus-producing lesion that does not communicate with the duct system, and it occurs most often in the body or tail of the pancreas (38). Also, MCNs contain cellular ovarian-type stroma that expresses hormone receptors as shown by immunohistochemistry. The 2015 Baltimore Consensus Conference revised the previous WHO classification of dysplasia into 3 levels, and recommended that pancreatic precancerous lesions (PanIN,
IPMN, and MCN) be divided into 2 levels (low-grade and high-grade), while middle-grade dysplasia in the original classification is classified as low-grade (39). The 5 th edition of the WHO digestive system tumor classification published in 2019 also changed the IPMN classification method to a secondary classification system, namely low-grade dysplasia (LGD) and high-grade dysplasia (HGD) (40) (Figure 2).

IPMN and invasive carcinoma can occur in the same pancreas. If the carcinoma arises in the area of the IPMN, it is designated as IPMN with associated invasive carcinoma. If the carcinoma is not contiguous with IPMN, it is designated as IPMN with a concomitant invasive carcinoma (21) (Figure 3). Invasive carcinoma can be divided into 2 types, namely colloid carcinoma and tubular (ductal) adenocarcinoma. Yamaguchi et al. found that IPMNs with associated invasive carcinoma are enriched for colloid carcinomas and main duct IPMNs, whereas IPMNs with a concomitant invasive carcinoma are almost always of the branch duct type, and the invasive carcinomas are typically tubular adenocarcinomas (41). IPMNs are considered to gradually develop from LGD to HGD and eventually into invasive carcinoma $(39,42,43)$. Approximately onethird of IPMNs are associated with an invasive carcinoma (4). Interestingly, a study using targeted next-generation sequencing methods showed that approximately $18 \%$ of invasive ductal adenocarcinomas co-occurring with IPMN are "possibly independent" because they do not share driving gene mutations (44). In another study, this kind of progression of IPMN into PDAC, i.e., PDAC which shares no driving gene mutations with IPMN, is known as the de novo subtype (45). There is a significant difference in the 5 -year survival rate of non-invasive and invasive IPMN patients (90\% and 50\%, respectively) (37). In a retrospective study of about 113 patients with IPMN, $65 \%$ of invasive IPMN patients relapsed, while only $8.3 \%$ of 73 noninvasive IPMN patients relapsed, and the 5-year survival rate of non-invasive IPMN patients was $88 \%$, while that of IPMN patients with related invasive cancer was 36\% (46). All pancreatic duct cells are at risk of developmental abnormalities, which are most typically manifested in patients with multifocal BD-IPMNs, and molecular analysis shows that most multifocal BD-IPMNs appear to arise independently, i.e., "clonal heterogeneity" (47). At present, the judgment of benign and malignant IPMN is very difficult. It is still challenging to accurately evaluate whether IPMN carries high-grade or invasive diseases that require surgical resection. A retrospective study showed that the combination of multiphase radiomic CT evaluation 

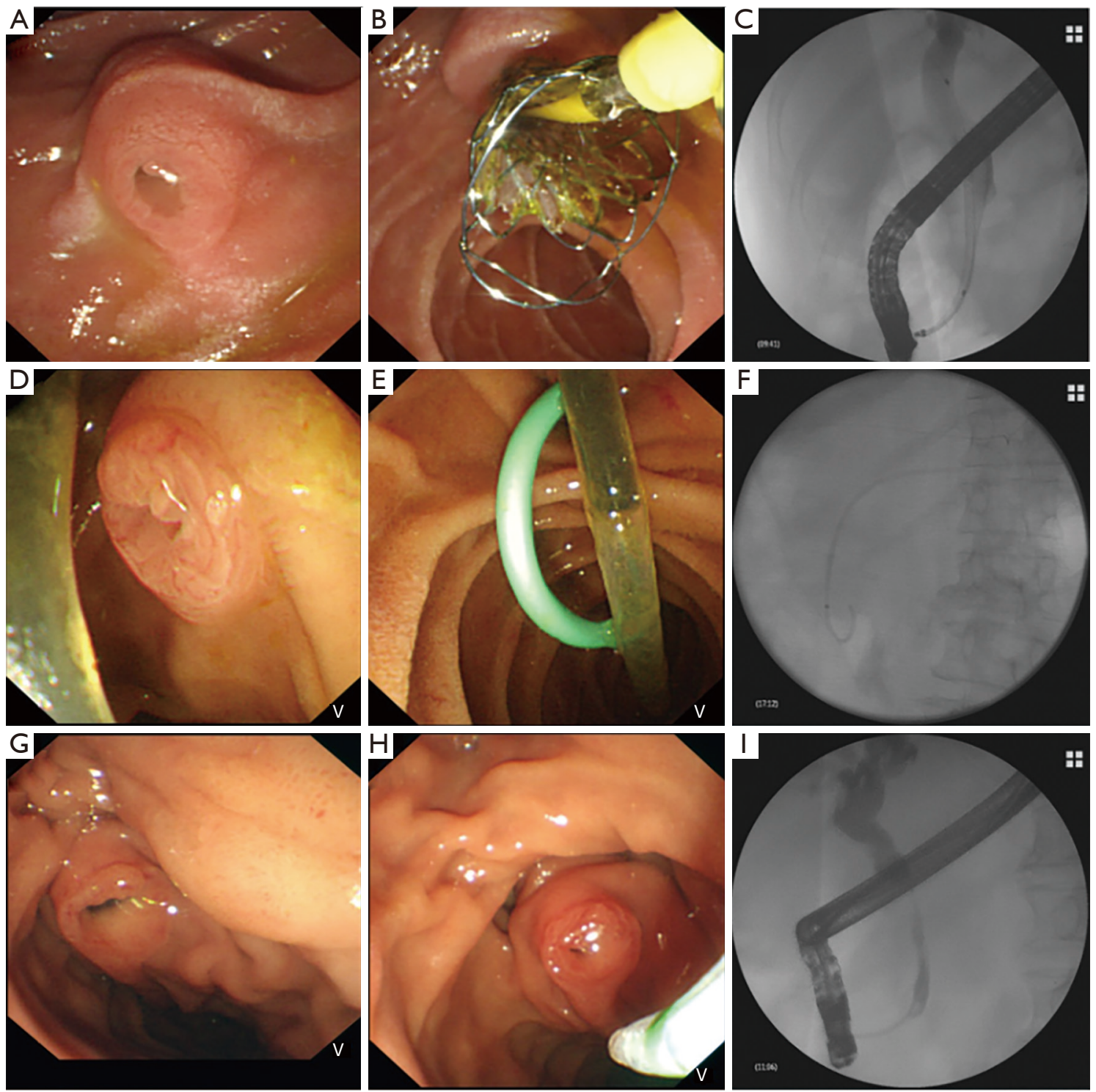

Figure 1 ERCP for IPMN. (A,B,C) A 78-year-old man with obstructive jaundice, considering IPMN-related invasive cancer, inoperable: (A) the large duodenal papilla is fish-mouthed with mucus overflow, (B) indwelling biliary stent, (C) biliary stent angiography. (D,E,F) A 64-year-old female, considering IPMN with acute pancreatitis, poor general condition, diabetes, coronary heart disease, inoperable: (D) the duodenal large papilla is chrysanthemum petal-like with yellow and white mucus overflow, $(\mathrm{E})$ indwelling pancreas tube stent, (F) pancreatic duct stent angiography. (G,H,I) A 50-year-old male, considering IPMN, the preoperative diagnosis is not clear, ERCP assists in the diagnosis: $(\mathrm{G})$ the duodenal large papilla is thickened like a sausage mouth with a large amount of mucus overflow, (H) ERCP examination, (I) cholangiography, the biliary tract is narrow. ERCP, endoscopic retrograde cholangiopancreatography; IPMN, intraductal papillary mucinous neoplasm.

and routine International Consensus Guidelines (ICG) (also called the Fukuoka Consensus Guidelines) standard analysis can improve the predictive ability of diagnosing the malignant degree of IPMN (48). A multi-institutional study showed that the proteins in pancreatic cyst fluid (CF) may help distinguish the degree of dysplasia of IPMN (49). In 2017, Permuth et al. found that an 8-lncRNA signature (including GLIS3-AS1, ADARB2-AS1, LINC00472, 


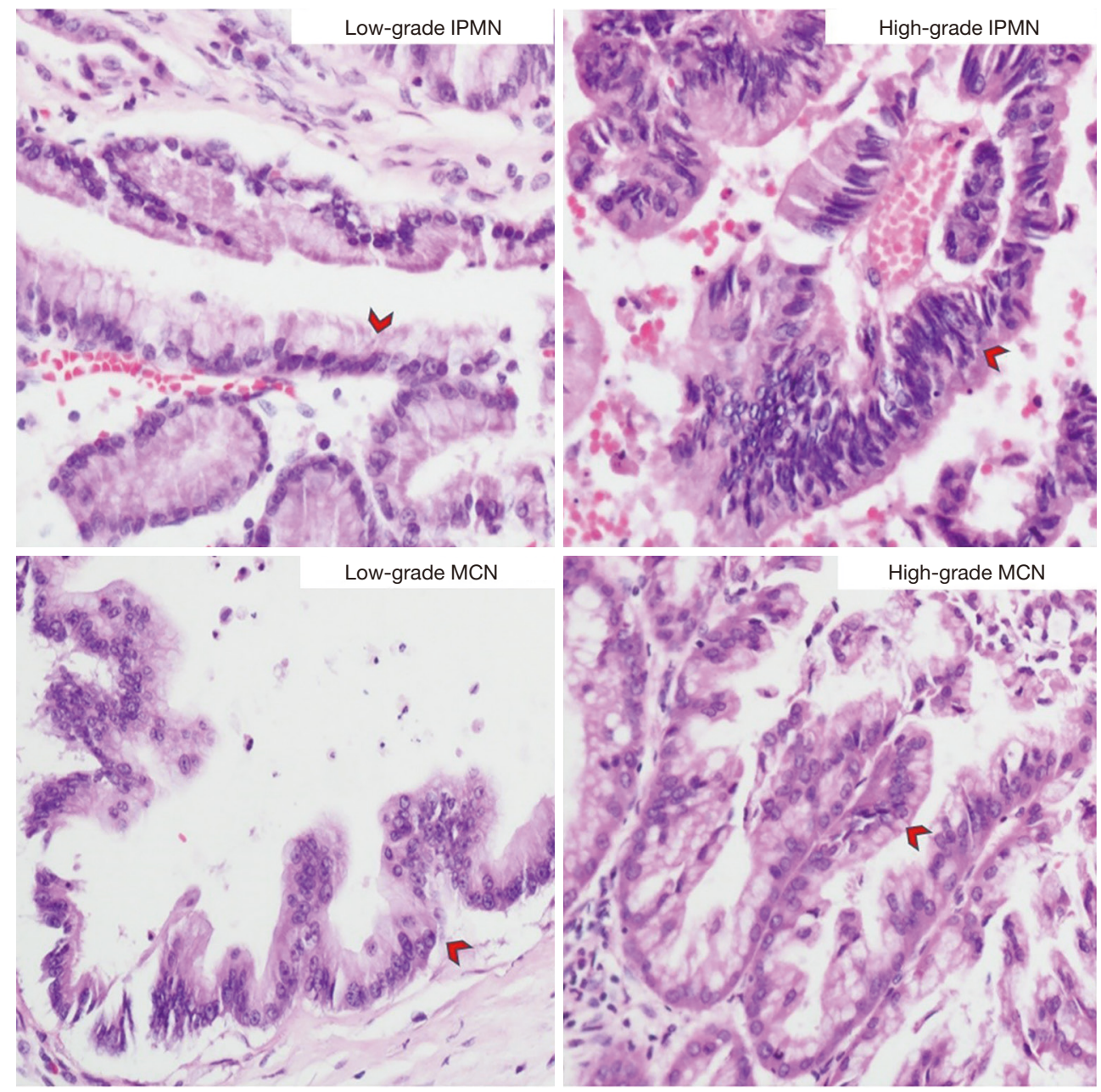

Figure 2 Low-grade and high-grade dysplasia of IPMN and MCN. Low-grade IPMNs are characterized by proliferation of columnar mucous cells in the duct and the absence of periductal mesenchyme, i.e., "ovarian-like mesenchyme". High-grade IPMNs are characterized by the structure and cells have obvious atypia. The structure of the papilla is more complex, with buds or branches visible. Low-grade MCNs are characterized by columnar epithelial cells with only mild structure and cell atypia. The nucleus is slightly enlarged and located at the base. High-grade MCNs are characterized by the structure and cells have obvious atypia. The papilla has disorderly branching and budding, the nucleus is double-layered, the polar direction disappears, pleomorphism is present, and nuclear division is common. Red arrows: typical performance described in the description text above. Hematoxylin and eosin staining, magnification $\times 200$.

ANRIL, PANDA, MEG3, PVT1, and UCA1) may contribute to the malignant prediction and diagnosis of IPMN (50). A study in 2021 showed that MUC5AC in circulating extracellular vesiclecan predict whether there is invasive carcinoma within IPMN, which may prevent unnecessary surgery (51).

According to cell morphology, IPMN can also be divided into gastric-type, intestinal-type, and pancreaticobiliarytype, and oncocytic-type IPMN is now recognized as a distinct entity, i.e. intraductal oncocytic papillary neoplasm
(IOPN) (21). Gastric-type IPMN is the most common type (approximately 70\% of cases). Gastric-type IPMN is composed of cells similar to gastric foveolae, usually with low-grade atypia, often occurring in pancreatic branch ducts, while intestinal IPMN often involves the main duct and several branch ducts (52). The pancreatobiliary subtype is the least common. It typically involves the main pancreatic duct and is characterized by high dysplasia (53). Colloid carcinomas of the pancreas are concurrent with intestinal-type IPMN, while ductal adenocarcinomas are 


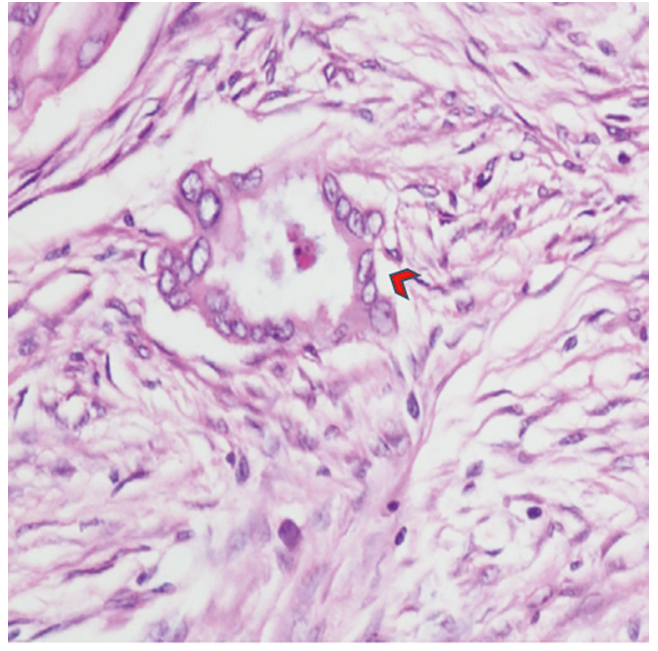

Figure 3 IPMN-related invasive carcinoma. Promote fibro interstitial hyperplasia helps to identify invasive carcinoma and trapped non-neoplastic glands. The infiltrating component resembles ductal adenocarcinoma, forming tubular and tubularlike structures. Red arrow: typical performance described in the description text above. Hematoxylin and eosin staining, magnification $\times 200$.

related to the gastric and pancreaticobiliary subtypes of IPMN (44). IOPN also grows in the pancreatic ducts, which is very similar to IPMN, but it contains eosinophils instead of columnar mucous cells. IOPN is a grossly cystic epithelial neoplasm composed of exophytic nodular projections lined by oncocytic glandular epithelium (54). Generally, lOPNs typically lack the alterations reported to be related to ductal adenocarcinoma and IPMN, such as mutations in KRAS, GNAS, and RNF43. In contrast, genes including ARHGAP26, ASXL1, EPHA8, and ERBB4 are recurrently mutated in some lOPNs (55). Based on both the architectural complexity and the degree of nuclear atypia, essentially all lOPNs have HGD (56). Recently, a retrospective study of 20 pancreatic IOPN patients found that PRKACA and PRKACB are important fusion genes related to IOPN, and these genes were not found in PCNs such as IPMNs (57). Intraductal tubulopapillary neoplasm (ITPN) is another type of pancreatic ductal tumor. ITPN of the pancreas is an intraductal, predominantly tubuleforming epithelial neoplasm without overt production of mucin. MUC5AC, a marker of all types of IPMNs, is almost never expressed in ITPNs.

\section{Surgical treatment}

In terms of postoperative pathology, the average incidence of invasive carcinoma and HGD in BD-IPMN is $18.5 \%$ and $31.1 \%$, respectively. The average incidence of invasive carcinoma and HGD in MD-IPMN is $43.1 \%$ and $61.6 \%$, respectively (26). Evidently, MD-IPMN is potentially more dangerous than BD-IPMN. Because of this, many guidelines believe that early surgery of MD-IPMN and MT-IPMN is necessary $(26,58,59)$. Interestingly, the malignant tumor with typical progression of BD-IPMN is tubular carcinoma, which is similar to typical PDAC and has a poor prognosis. The typical progressive malignant tumor of MD-IPMN is colloid carcinoma, which is characterized by abundant extracellular mucins and scattered cancerous epithelium, and has a better prognosis.

At present, early surgery is still recognized as the most effective management approach for IPMN. It is often difficult to assess the degree of IPMN dysplasia before and during surgery, and surgery is the only way to cure the disease. Therefore, removing the majority of these potentially malignant neoplasms are necessary. However, the indications for surgery are inconsistent between different guidelines. For example, the Fukuoka guidelines specify that enhancing mural nodules $>5 \mathrm{~mm}$, MPD $>10 \mathrm{~mm}$, and jaundice are high-risk stigmata for surgery. When a patient meets any of these items and can tolerate surgery, surgery should be performed, and cyst growth $\geq 5 \mathrm{~mm} / 2$ years, cyst dimension $\geq 3 \mathrm{~cm}$, thickening of the cyst wall, abrupt pancreatic duct caliber change, increase of serum carbohydrate antigen (CA) 19-9, and pancreatitis should be used as "worrisome features" for evaluating whether to operate (26). However, the European guidelines specify that surgery should be performed when the main pancreatic duct $>5 \mathrm{~mm}$, and the malignant cytology results are the absolute indications for surgery (58). Relative indications are cyst growth $\geq 5 \mathrm{~mm} /$ year, cyst dimension $\geq 4 \mathrm{~cm}$, serum CA 19-9 $\geq 37 \mathrm{U} / \mathrm{mL}$, newly-onset diabetes, and acute pancreatitis, which are stricter than the Japanese guidelines. Jan $e t$ al. verified the effectiveness of increased serum CA 19-9 and newly-onset diabetes as indications for surgery through an evidence-based method (60).

In view of the high malignant transformation rate of MTIPMN and MD-IPMN, it is recommended that all patients suitable for surgery should be treated with surgery. The surgical method is determined based on the extent of the 
lesions shown by the imaging examination results. Because most IPMN occurs in the head of the pancreas, the most common surgical method is pancreaticoduodenectomy. Local pancreatectomy is also feasible for IPMN with focal lesions, but it is recommended to obtain frozen sections of the cutting edge during the operation to guide whether to expand the scope of resection. When multifocal lesions are involved, each lesion needs to be evaluated separately, and then the surgical method is formulated. In the case of increased probability of malignant transformation, such as all MPD involvement, familial pancreatic cancer and mural nodules, total pancreatectomy is required (61). Laparoscopic resection of the pancreas body and tail with or without spleen preservation has been widely used. During the operation, the surgical margin should be paid attention to. If it is positive, the resection range should be increased. De-epithelization should not be regarded as a negative margin. The pancreatic duct epithelium of the resection margin must be normal epithelium.

It is worth noting that although many guidelines have provided their own recommendations, they are still not enough to develop the best treatment for patients. For example, in patients with dilated main pancreatic duct, it is necessary to conduct further evaluation, as the main pancreatic duct may be dilated for other reasons, such as chronic pancreatitis, or, for example, the presence and organization of wall nodules. There is a strong link between pathologic grades (62), but this does not mean that flat lesions have a very low rate of malignant transformation. Long-term follow-up is still necessary (63). By developing a medical natural language processing system (NLP) for functional registration of IPMN patients, the identification and follow-up of IPMN patients can be promoted (64).

\section{Postoperative follow-up}

IPMNs are present for many years before they develop into invasive cancer. Early detection and intervention of IPMN may be beneficial to patients, and early surveillance of IPMN provides an opportunity to reduce cancer deaths associated with pancreatic cancer (65). Recently, a large long-term study of 1,404 patients with BD-IPMNs found that the 5-year incidence of pancreatic cancer was $3.3 \%$ after the diagnosis of IPMN, while the 15 -year incidence was $15.0 \%(66)$. IPMNs are often multifocal. For the remnant pancreas after the resection of an IPMN, there is the risk of progression of pre-existing IPMNs, the risk of developing new IPMNs, and a risk of developing pancreatic cancer unassociated with an IPMN in the pancreas. The risk of IPMN progression after resection will not diminish over time. As long as the patient is still suitable for surgery, surveillance should continue indefinitely (26). The American College of Gastroenterology (ACG) Clinical Guideline strongly recommends postoperative surveillance for all patients undergoing surgical resection (38). The Fukuoka Consensus Guidelines also recommend that all IPMN patients, even those with non-invasive IPMN who have negative surgical margins, should be surveilled after resection, in order to detect the development of new IPMNs that require surgery or concomitant PDAC (26). A study showed that the 3 most common features associated with the progression of pancreatic remnants are "margin positive resection", the presence of HGD in resected specimens, and a family history of PDAC (67). A retrospective study spanning nearly 20 years showed that patients with MDIPMN who undergo segmental pancreatectomy are at a higher risk of subsequent pancreatic cancer if they are found to have HGD or invasive cancer during resection, and they may gain benefits from post-operative follow-up (68). Patients who have evidence of IPMN-associated invasive carcinoma have the same follow-up strategy as those who have a resected pancreatic cancer (26). Monitoring must strictly follow the guidelines. The European guidelines recommend that patients who do not meet the criteria for surgical intervention should be followed up regularly, including a 6-month follow-up in the first year, and then once a year until the risk factor disappears. For patients with relative indications for surgery but not undergoing surgery, patients undergoing surgery due to LGD, the elderly, and patients with severe complications, follow-up should be performed twice a year. For patients who have undergone surgery, lifelong follow-up is recommended for MD-IPMN. Patients with HGD should be closely followed up every 6 months for the first 2 years, and then monitored annually (58). The current follow-up examinations mostly use CT and EUS. The guidelines of the American Gastroenterological Association (AGA) are the only guidelines that recommend stopping surveillance for pancreatic cysts. They recommend that if there are no high-risk features and the cyst size is stable, surveillance should be stopped after 5 years (69). For patients without mixed IPMN and without a strong family history of pancreatic carcinoma, the AGA also recommends against routine surveillance of pancreatic cysts without HGD or malignancy during surgical resection (69). However, there is still controversy regarding stopping IPMN surveillance 
in the ACG guidelines (70). Large, prospective, multicenter studies are needed to provide evidence to help future guidelines.

\section{Conclusions}

IPMN is one of the most important precancerous lesions of pancreatic carcinoma, and the knowledge and management of IPMN is also critical. IPMN is now classified into 2 tiers of dysplasia (low-grade and high-grade), and is also divided into gastric-type, intestinal-type, pancreaticobiliarytype, and oncocytic-type IPMN, with the latter type now being recognized as a distinct entity, namely IOPN. With the development of imaging, the detection rate of IPMN has been greatly improved. However, its degree of malignancy is still difficult to assess. Symptomatic IPMNs overall have a higher risk of HGD and invasive carcinoma. According to the positional relationship between IPMN and invasive cancer in the pancreas, $\mathrm{WHO}$ divides invasive carcinoma into 2 types, namely IPMN with associated invasive carcinoma and IPMN with a concomitant invasive carcinoma. The microscopic relationship between IPMN and invasive cancer is not so simple. Gross, radiologic, and microscopic findings, as well as genetic studies should be incorporated to produce evidence to distinguish the 2 types. The ICG (also called the Fukuoka Consensus Guidelines) is based on worrisome features and high-risk stigmata to decide between resection or further evaluation for IPMN. Postoperative follow-up is also crucial for IPMN. In the remnant pancreas, regardless of whether disease is caused by the growth of a separate lesion or recurrence of the patient's original IPMN, careful clinical follow-up is required after resection for IPMN patients with any degree of dysplasia and any marginal state. The available guidelines lack strong evidence for IPMN management, as they mainly rely on expert opinions. Thus, large, prospective, multicenter studies and randomized clinical trials are required. The management of IPMN requires guidelines that can better predict the recurrence and tumor malignancy of IPMN, and the treatment of IPMN should be based on patient and tumor characteristics and multidisciplinary discussion.

\section{Acknowledgments}

Funding: Liaoning Provincial Department of Education Science Research Project (No. L2014299) and National Natural Science Foundation of China (No. 81572360).

\section{Footnote}

Reporting Checklist: The authors have completed the Narrative Review reporting checklist. Available at https:// dx.doi.org/10.21037/gs-21-450

Conflicts of Interest: All authors have completed the ICMJE uniform disclosure form (available at https://dx.doi. org/10.21037/gs-21-450). The authors report funding support from the Liaoning Provincial Department of Education Science Research Project (No. L2014299) and National Natural Science Foundation of China (No. $81572360)$. The authors have no other conflicts of interest to declare.

Ethical Statement: The authors are accountable for all aspects of the work in ensuring that questions related to the accuracy or integrity of any part of the work are appropriately investigated and resolved.

Open Access Statement: This is an Open Access article distributed in accordance with the Creative Commons Attribution-NonCommercial-NoDerivs 4.0 International License (CC BY-NC-ND 4.0), which permits the noncommercial replication and distribution of the article with the strict proviso that no changes or edits are made and the original work is properly cited (including links to both the formal publication through the relevant DOI and the license). See: https://creativecommons.org/licenses/by-nc-nd/4.0/.

\section{References}

1. Rahib L, Smith BD, Aizenberg R, et al. Projecting cancer incidence and deaths to 2030: the unexpected burden of thyroid, liver, and pancreas cancers in the United States. Cancer Res 2014;74:2913-21.

2. Bray F, Ferlay J, Soerjomataram I, et al. Global cancer statistics 2018: GLOBOCAN estimates of incidence and mortality worldwide for 36 cancers in 185 countries. CA Cancer J Clin 2018;68:394-424.

3. Abdelkader A, Hunt B, Hartley CP, et al. Cystic Lesions of the Pancreas: Differential Diagnosis and Cytologic-Histologic Correlation. Arch Pathol Lab Med 2020;144:47-61.

4. Matthaei H, Schulick RD, Hruban RH, et al. Cystic precursors to invasive pancreatic cancer. Nat Rev Gastroenterol Hepatol 2011;8:141-50. 
5. Aronsson L, Marinko S, Ansari D, et al. Adjuvant therapy in invasive intraductal papillary mucinous neoplasm (IPMN) of the pancreas: a systematic review. Ann Transl Med 2019;7:689.

6. Fritz S, Fernandez-del Castillo C, Mino-Kenudson M, et al. Global genomic analysis of intraductal papillary mucinous neoplasms of the pancreas reveals significant molecular differences compared to ductal adenocarcinoma. Ann Surg 2009;249:440-7.

7. Lee JH, Kim Y, Choi JW, et al. KRAS, GNAS, and RNF43 mutations in intraductal papillary mucinous neoplasm of the pancreas: a meta-analysis. Springerplus 2016;5:1172.

8. Huang B, Trujillo MA, Fujikura K, et al. Molecular characterization of organoids derived from pancreatic intraductal papillary mucinous neoplasms. J Pathol 2020;252:252-62.

9. Zamboni G, Hirabayashi K, Castelli P, et al. Precancerous lesions of the pancreas. Best Pract Res Clin Gastroenterol 2013;27:299-322.

10. Collet L, Ghurburrun E, Meyers N, et al. Kras and Lkb1 mutations synergistically induce intraductal papillary mucinous neoplasm derived from pancreatic duct cells. Gut 2020;69:704-14.

11. Valsangkar NP, Morales-Oyarvide V, Thayer SP, et al. 851 resected cystic tumors of the pancreas: a 33-year experience at the Massachusetts General Hospital. Surgery 2012;152:S4-12.

12. Jin C, Li J, Zou C, et al. Lymph node ratio predicts prognosis in patients with surgically resected invasive pancreatic cystic neoplasms. Transl Cancer Res 2020;9:5843-56.

13. Kosmahl M, Pauser U, Peters K, et al. Cystic neoplasms of the pancreas and tumor-like lesions with cystic features: a review of 418 cases and a classification proposal. Virchows Archiv 2004;445:168-78.

14. Yoon WJ, Lee JK, Lee KH, et al. Cystic Neoplasms of the Exocrine Pancreas: An Update of a Nationwide Survey in Korea. Pancreas 2008;37:254-8.

15. Chaudhari VA, Pradeep R, Ramesh H, et al. Surgery for cystic tumors of pancreas: Report of high-volume, multicenter Indian experience over a decade. Surgery 2019;166:1011-6.

16. Rezaee N, Khalifian S, Cameron JL, et al. Smoking is not associated with severe dysplasia or invasive carcinoma in resected intraductal papillary mucinous neoplasms. J Gastrointest Surg 2015;19:656-65.

17. Nakagawa T, Masuda A, Toyama H, et al. Smoking Status and the Incidence of Pancreatic Cancer Concomitant
With Intraductal Papillary Mucinous Neoplasm. Pancreas 2017;46:582-8.

18. Carr RA, Roch AM, Shaffer K, et al. Smoking and IPMN malignant progression. Am J Surg 2017;213:494-7.

19. Del Chiaro M, Valente R, Wolfgang C. Response to Comment on "Main Duct Dilatation is the Best Predictor of High-grade Dysplasia or Invasion in Intraductal Papillary Mucinous Neoplasms of the Pancreas". Ann Surg 2019;270:e109-10.

20. Chang YR, Park JK, Jang JY, et al. Incidental pancreatic cystic neoplasms in an asymptomatic healthy population of 21,745 individuals: Large-scale, single-center cohort study. Medicine (Baltimore) 2016;95:e5535.

21. Board WCTE. Digestive System Tumours. International Agency for Research on Cancer; 2019.

22. Maguchi H, Tanno S, Mizuno N, et al. Natural History of Branch Duct Intraductal Papillary Mucinous Neoplasms of the Pancreas: A Multicenter Study in Japan. Pancreas 2011;40:364-70.

23. Salvia R, Fernandez-del Castillo C, Bassi C, et al. Mainduct intraductal papillary mucinous neoplasms of the pancreas: clinical predictors of malignancy and long-term survival following resection. Ann Surg 2004;239:678-85; discussion 685-7.

24. Moriya T, Hashimoto Y, Traverso LW. The duration of symptoms predicts the presence of malignancy in 210 resected cases of pancreatic intraductal papillary mucinous neoplasms. J Gastrointest Surg 2011;15:76270; discussion 770-1.

25. Okabayashi $T$, Shima $Y$, Kosaki $T$, et al. Invasive carcinoma derived from branch duct-type IPMN may be a more aggressive neoplasm than that derived from main ducttype IPMN. Oncol Lett 2013;5:1819-25.

26. Tanaka M, Fernandez-Del Castillo C, Kamisawa T, et al. Revisions of international consensus Fukuoka guidelines for the management of IPMN of the pancreas. Pancreatology 2017;17:738-53.

27. Sodickson A, Baeyens PF, Andriole KP, et al. Recurrent CT, Cumulative Radiation Exposure, and Associated Radiation-induced Cancer Risks from CT of Adults. Radiology 2009;251:175-84.

28. Ohno E, Hirooka Y, Itoh A, et al. Intraductal Papillary Mucinous Neoplasms of the Pancreas: Differentiation of Malignant and Benign Tumors by Endoscopic Ultrasound Findings of Mural Nodules. Ann Surg 2009;249:628-34.

29. Uribarri-Gonzalez L, Keane MG, Pereira SP, et al. Agreement among Magnetic Resonance Imaging/Magnetic Resonance Cholangiopancreatography (MRI-MRCP) 
and Endoscopic Ultrasound (EUS) in the evaluation of morphological features of Branch Duct Intraductal Papillary Mucinous Neoplasm (BD-IPMN). Pancreatology 2018;18:170-5.

30. Hwang J, Kim YK, Min JH, et al. Comparison between MRI with MR cholangiopancreatography and endoscopic ultrasonography for differentiating malignant from benign mucinous neoplasms of the pancreas. Eur Radiol 2018;28:179-87.

31. Choi S-Y, Kim JH, Yu MH, et al. Diagnostic performance and imaging features for predicting the malignant potential of intraductal papillary mucinous neoplasm of the pancreas: a comparison of EUS, contrast-enhanced CT and MRI. Abdom Radiol (NY) 2017;42:1449-58.

32. Yoshioka T, Shigekawa M, Yamai T, et al. The safety and benefit of pancreatic juice cytology under ERCP in IPMN patients. Pancreatology 2016;16:1020-7.

33. Wu J, Matthaei H, Maitra A, et al. Recurrent GNAS mutations define an unexpected pathway for pancreatic cyst development. Sci Transl Med 2011;3:92ra66.

34. Singhi AD, Nikiforova MN, Fasanella KE, et al. Preoperative GNAS and KRAS testing in the diagnosis of pancreatic mucinous cysts. Clin Cancer Res 2014;20:4381-9.

35. Frampton AE, Stebbing J, Gall TM, et al. Activating mutations of GNAS and KRAS in cystic fluid can help detect intraductal papillary mucinous neoplasms of the pancreas. Expert Rev Mol Diagn 2015;15:325-8.

36. Sugiyama M, Atomi Y. Intraductal Papillary Mucinous Tumors of the Pancreas: Imaging Studies and Treatment Strategies. Ann Surg 1998;228:685-91.

37. Crippa S, Fernandez-Del Castillo C, Salvia R, et al. Mucin-producing neoplasms of the pancreas: an analysis of distinguishing clinical and epidemiologic characteristics. Clin Gastroenterol Hepatol 2010;8:213-9.

38. Elta GH, Enestvedt BK, Sauer BG, et al. ACG Clinical Guideline: Diagnosis and Management of Pancreatic Cysts. Am J Gastroenterol 2018;113:464-79.

39. Basturk O, Hong S-M, Wood LD, et al. A Revised Classification System and Recommendations From the Baltimore Consensus Meeting for Neoplastic Precursor Lesions in the Pancreas. Am J Surg Pathol 2015;39:1730-41.

40. Nagtegaal ID, Odze RD, Klimstra D, et al. The 2019 WHO classification of tumours of the digestive system. Histopathology 2020;76:182-8.

41. Yamaguchi K, Kanemitsu S, Hatori T, et al. Pancreatic Ductal Adenocarcinoma Derived From IPMN and
Pancreatic Ductal Adenocarcinoma Concomitant With IPMN. Pancreas 2011;40:571-80.

42. Maitra A, Fukushima N, Takaori K, et al. Precursors to Invasive Pancreatic Cancer. Adv Anat Pathol 2005;12:81-91.

43. Lennon AM, Wolfgang C. Cystic Neoplasms of the Pancreas. J Gastrointest Surg 2013;17:645-53.

44. Felsenstein M, Noë M, Masica DL, et al. IPMNs with co-occurring invasive cancers: neighbours but not always relatives. Gut 2018;67:1652.

45. Omori Y, Ono Y, Tanino M, et al. Pathways of Progression From Intraductal Papillary Mucinous Neoplasm to Pancreatic Ductal Adenocarcinoma Based on Molecular Features. Gastroenterology 2019;156:647-61.e2.

46. Chari ST, Yadav D, Smyrk TC, et al. Study of recurrence after surgical resection of intraductal papillary mucinous neoplasm of the pancreas. Gastroenterology 2002;123:1500-7.

47. Matthaei H, Norris AL, Tsiatis AC, et al. Clinicopathological Characteristics and Molecular Analyses of Multifocal Intraductal Papillary Mucinous Neoplasms of the Pancreas. Ann Surg 2012;255:326-33.

48. Polk SL, Choi JW, McGettigan MJ, et al. Multiphase computed tomography radiomics of pancreatic intraductal papillary mucinous neoplasms to predict malignancy. World J Gastroenterol 2020;26:3458.

49. Al Efishat MA, Attiyeh MA, Eaton AA, et al. Multiinstitutional Validation Study of Pancreatic Cyst Fluid Protein Analysis for Prediction of High-risk Intraductal Papillary Mucinous Neoplasms of the Pancreas. Ann Surg 2018;268:340-7.

50. Permuth JB, Chen DT, Yoder SJ, et al. Linc-ing Circulating Long Non-coding RNAs to the Diagnosis and Malignant Prediction of Intraductal Papillary Mucinous Neoplasms of the Pancreas. Sci Rep 2017;7:10484.

51. Yang KS, Ciprani D, O'Shea A, et al. Extracellular Vesicle Analysis Allows for Identification of Invasive IPMN. Gastroenterology 2021;160:1345-58.e11.

52. Furukawa T, Klöppel G, Volkan Adsay N, et al. Classification of types of intraductal papillary-mucinous neoplasm of the pancreas: a consensus study. Virchows Archiv 2005;447:794-9.

53. Shimizu T, Akita M, Sofue K, et al. Pancreatobiliary-type intraductal papillary mucinous neoplasm of the pancreas may have 2 subtypes with distinct clinicopathologic and genetic features. Hum Pathol 2019;91:26-35.

54. Adsay NV, Adair CF, Heffess CS, et al. Intraductal Oncocytic Papillary Neoplasms of the Pancreas. Am J Surg Pathol 1996;20:980-94. 
55. Basturk O, Tan M, Bhanot U, et al. The oncocytic subtype is genetically distinct from other pancreatic intraductal papillary mucinous neoplasm subtypes. Mod Pathol 2016;29:1058-69.

56. Kallen ME, Naini BV. Intraductal Oncocytic Papillary Neoplasms of the Pancreas. Arch Pathol Lab Med 2016;140:992-6.

57. Singhi AD, Wood LD, Parks E, et al. Recurrent Rearrangements in PRKACA and PRKACB in Intraductal Oncocytic Papillary Neoplasms of the Pancreas and Bile Duct. Gastroenterology 2020;158:573-82.e2.

58. European Study Group on Cystic Tumours of the Pancreas. European evidence-based guidelines on pancreatic cystic neoplasms. Gut 2018;67:789-804.

59. Scheiman JM, Hwang JH, Moayyedi P. American Gastroenterological Association Technical Review on the Diagnosis and Management of Asymptomatic Neoplastic Pancreatic Cysts. Gastroenterology 2015;148:824-48.e22.

60. Jan IS, Chang MC, Yang CY, et al. Validation of Indications for Surgery of European Evidence-Based Guidelines for Patients with Pancreatic Intraductal Papillary Mucinous Neoplasms. J Gastrointest Surg 2020;24:2536-43.

61. Cortegoso Valdivia P, Chiala C, Venezia L, et al. Diagnosis and management of intraductal papillary mucinous neoplasms of the pancreas. Acta Biomed 2018;89:147-52.

62. Lee JE, Choi SY, Min JH, et al. Determining Malignant Potential of Intraductal Papillary Mutinous Neoplasm of the Pancreas: CT versus MRI by Using Revised 2017 International Consensus Guidelines. Radiology 2019;293:134-43.

63. Koshita S, Fujita N, Noda Y, et al. Invasive carcinoma derived from "flat type" branch duct intraductal

Cite this article as: Ma G, Li G, Xiao Z, Gou A, Xu Y, Song S, Guo K, Liu Z. Narrative review of intraductal papillary mucinous neoplasms: pathogenesis, diagnosis, and treatment of a true precancerous lesion. Gland Surg 2021;10(7):2313-2324. doi: $10.21037 / g s-21-450$ papillary mucinous neoplasms of the pancreas: impact of classification according to the height of mural nodule on endoscopic ultrasonography. J Hepatobiliary Pancreat Sci 2015;22:301-9.

64. Al-Haddad MA, Friedlin J, Kesterson J, et al. Natural language processing for the development of a clinical registry: a validation study in intraductal papillary mucinous neoplasms. HPB (Oxford) 2010;12:688-95.

65. Lennon AM, Wolfgang CL, Canto MI, et al. The Early Detection of Pancreatic Cancer: What Will It Take to Diagnose and Treat Curable Pancreatic Neoplasia? Cancer Res 2014;74:3381.

66. Oyama H, Tada M, Takagi K, et al. Long-term Risk of Malignancy in Branch-Duct Intraductal Papillary Mucinous Neoplasms. Gastroenterology 2020;158:226-37.e5.

67. He J, Cameron JL, Ahuja N, et al. Is It Necessary to Follow Patients after Resection of a Benign Pancreatic Intraductal Papillary Mucinous Neoplasm? J Am Coll Surg 2013;216:657-65.

68. Majumder S, Philip NA, Singh Nagpal SJ, et al. HighGrade Dysplasia in Resected Main-Duct Intraductal Papillary Mucinous Neoplasm (MD-IPMN) is Associated with an Increased Risk of Subsequent Pancreatic Cancer. Am J Gastroenterol 2019;114:524-9.

69. Vege SS, Ziring B, Jain R, et al. American gastroenterological association institute guideline on the diagnosis and management of asymptomatic neoplastic pancreatic cysts. Gastroenterology 2015;148:819-22; quize 812-3.

70. Farrell JJ. Editorial: Stopping Pancreatic Cyst Surveillance? Am J Gastroenterol 2017;112:1162-4.

(English Language Editor: C Betlzar) 